\title{
Prostate Imaging Reporting and Data System in prostate cancer staging and planning for radical prostatectomy
}

\author{
Mieszko Kozikowski ${ }^{1}$, Bartłomiej Zagożdżon ${ }^{1}$, Magdalena Gola², Jakub Dobruch ${ }^{1}$ \\ ${ }^{1}$ Department of Urology, Centre of Postgraduate Medical Education, Warsaw, Poland \\ ${ }^{2}$ Department of Diagnostic Imaging, European Health Centre, Otwock, Poland
}

Videosurgery Miniinv 2019; 14 (2): 262-270

DOI: https://doi.org/10.5114/wiitm.2019.83869

\begin{abstract}
Introduction: The Prostate Imaging Reporting and Data System (PI-RADS) was mainly developed for the purposes of prostate cancer $(P C a)$ detection. However, its widespread use suggests that it may play a role in a preoperative workup prior to endoscopic radical prostatectomy (ERP).

Aim: To evaluate the prognostic value of PI-RADS in predicting extraprostatic extension (EPE) and its influence on surgical planning of ERP.

Material and methods: The analysis involved data of 154 consecutive prostate cancer patients, in whom multiparametric 3.0T magnetic resonance imaging (mpMRI) was performed before ERP. Standard descriptive assessment of mPMRI images was compared with the PI-RADS system with respect to prostate cancer staging and subsequent potential surgical template adjustment.

Results: PI-RADS significantly outperformed the standard way of mpMRI reporting in staging (AUC $=0.615$ vs. 0.552 , $p=0.036)$ with PI-RADS 5 established as the best threshold. After reevaluation of imaging, the initial surgical plan was modified based on $\mathrm{mPMRI}$ in terms of feasibility and extent of neurovascular bundle preservation during ERP on 96 (31.2\%) sides, while on the remaining 212 (68.8\%) sides the templates were left unchanged. Decisions based on $\mathrm{mPMRI}$ were not associated with increased risk of a positive surgical margin (PSM).

Conclusions: The PI-RADS outperforms the standard staging method using mpMRI and may assist the decision-making process regarding the extent of resection during ERP without increasing the risk of PSM.
\end{abstract}

Key words: radical prostatectomy, decision-making, neurovascular bundle, multiparametric magnetic resonance imaging, prostate cancer, mini-invasive surgery.

\section{Introduction}

Local prostate cancer ( $\mathrm{PCa}$ ) staging has a considerable impact on the extent of radical prostatectomy (RP) template. Magnetic resonance imaging (MRI) has been increasingly used in this context, although with rather modest results so far [1]. Various reasons for limited staging performance of multiparametric magnetic resonance imaging (mpMRI) were identified, including limited ability in the recogni- tion of focal extraprostatic infiltration by only tiny abnormal PCa glands [2]. Nevertheless, mpMRI has been proven to exert a considerable impact on the decision-making process prior to surgery regarding neurovascular bundle (NVB) preservation in nearly half of the cases [3]. Minimally invasive techniques that allow NVB sparing are gaining interest due to growing awareness of postoperative functional outcomes in terms of continence and erectile function and yet the possibility of providing negative surgical

\section{Address for correspondence}

Mieszko Kozikowski MD, Department of Urology, Centre of Postgraduate Medical Education, European Health Centre - Otwock,

14/18 Borowa St, 05-400 Otwock, Poland, phone: +48 792272 890, e-mail: mieszkokozikowski9@gmail.com 
margins, underscoring the need for individually tailored surgery in the era of mpMRI [4].

Since the European Society of Urogenital Radiology (ESUR) introduced Prostate Imaging-Reporting and Data System version 1 (PI-RADSv1) in 2012, it has gained widespread use in PCa diagnostics [5]. This system uses scoring of magnetic resonance images to assess the likelihood of detecting significant PCa [6]. Although the Prostate Imaging-Reporting and Data System (PI-RADS) was designed mainly for detection purposes, clinicians found other various applications beyond its designated function [7]. Radiologic findings, when converted into the PI-RADS scoring system, can have a role as a surrogate marker for pathological features after $\mathrm{RP}$ or even biochemical recurrence (BCR) [8]. The system may also serve as a strong predictor of extracapsular extension (ECE), therefore reducing the risk of understaging and facilitating precise preoperative planning [9].

\section{Aim}

We sought to evaluate the influence of PI-RADS version 2 (PI-RADSv2) in comparison with standard $\mathrm{PCa}$ staging with $\mathrm{MRI}$ on preoperative planning in men subjected to endoscopic radical prostatectomy (ERP).

\section{Material and methods}

\section{Population}

The prospectively studied cohort comprised 154 consecutive patients diagnosed with PCa who underwent ERP with preoperative 3.0 Tesla mpMRI done between February 2015 and August 2017 for staging purposes. The study cohort was different from that published in our previous study [10]. Imaging was performed at least 6 weeks after prostate biopsy following ESUR guidelines [6]. The images were reevaluated before surgery to establish the template of resection with special attention to location of lesion, PI-RADS, iTNM (radiologic stage) and proximity to NVB. Feasibility and extent of NVB sparing surgery were determined as well. In all cases, the same surgical team responsible for subsequent ERP participated in reevaluation of $\mathrm{mpMRI}$ images. The study protocol was approved by the local ethics committee and informed consent was taken before the surgery in all cases.

\section{Multiparametric magnetic resonance imaging}

mpMRI was performed in all cases with an Achieva 3.0-T MRI TX (Philips, Amsterdam, The Netherlands) with dual RF transmitter and 32 independent receiving channels. The MRI protocol included: axial T2-weighted turbo spin echo sequence, axial diffusion-weighted imaging (DWI) spin echo sequence with apparent diffusion coefficient map, axial dynamic contrast-enhanced imaging, axial T1-weighted spin echo with selective fat suppression sequence, axial T1-weighted turbo field echo sequence, coronal and sagittal T2-weighted turbo spin echo sequence.

The images were interpreted by a single experienced radiologist specialized in genitourinary tract diagnostics, who was not blinded to clinical characteristics including serum prostate-specific antigen (PSA), digital rectal examination (DRE) or biopsy results. The following criteria suspicious for extraprostatic extension (EPE) in imaging were adopted: irregular shape of prostatic capsule, NVB infiltration, seminal vesicle invasion, obliteration of rectoprostatic angle, overt extraprostatic tumor, periprostatic fat infiltration, bladder neck invasion or infiltration of rectum wall. The PI-RADS system was used to score identified lesions according to ESUR recommendations $[5,6]$. In this system a score from 1 to 5 is assigned to suspicious lesions identified in the gland based on specific radiologic signs found in multiple MRI sequences. The score reflects the likelihood of evaluated foci harboring significant PCa. Of note, lesions with definite EPE or invasive appearance are a priori considered PI-RADS 5. Finally, mpMRI was reevaluated by the surgical team in cooperation with the radiologist just before ERP to individualize the approach with respect to the surgical template that involved NVB preservation.

\section{Endoscopic radical prostatectomy}

Endoscopic radical prostatectomy was carried out by two surgeons having significant experience in oncological and endoscopic surgery. The images and clinical data were reevaluated before surgery to establish the template of resection with special attention to location of lesion, PI-RADS, iTNM (radiologic stage) and proximity to NVB. Feasibility and extent of NVB sparing surgery were determined considering preoperative clinical characteristics in 
connection with MRI images. NVBs were preserved separately on both sides with either an interfascial or an intrafascial approach. No preservation was implemented if mpMRI suggested EPE or a PI-RADS 5 tumor focus adjacent to the NVB on the ipsilateral side. Otherwise, the decision about NVB preservation was made based on clinical and imaging characteristics including PI-RADS score. For example, we considered NVB sparing possible on a side with a PI-RADS 3 or PI-RADS 4 lesion, unless other clinical variables were highly suspicious for the presence of locally advanced disease (EPE).

In low-risk cases - PCa cT $\leq 2 \mathrm{a}, \mathrm{GS}<7$ in biopsy and PSA below $10 \mathrm{ng} / \mathrm{ml}$ - extraperitoneal ERP was performed. Otherwise patients underwent transperitoneal ERP with extended pelvic lymph node dissection as previously described [11]. In uncertain cases we referred to Briganti's nomogram predicting lymph node involvement [12]. If the calculated risk of nodal metastases was equal to or greater than $5 \%$, extended pelvic lymph node dissection was performed during ERP.

\section{Histopathological examination}

The specimens were examined by a pathologist specialized in urooncology. The report was reevaluated by another experienced uropathologist from the urological faculty in case of adverse pathology: presence of EPE or positive surgical margins (PSM). The histopathological report typically included: prostate volume, type of cancer, Gleason score, tumor localization and extension, surgical margin status, number of resected and positive nodes. Following the European Association of Urology (EAU) guidelines EPE was defined as the presence of tumor beyond the prostate capsule and was substaged into extracapsular extension and seminal vesicle invasion. Surgical margins were positive if cancer cells were found within the inked surface of the prostate specimen. The locations of EPE and PSMs were registered and analyzed to determine whether the modification of the surgical template after mpMRI reevaluation was responsible for the PSM. The appropriateness of surgical plan change was assessed in per side analysis and was based on the presence of ECE or PSM in the proximity to NVB at histopathological examination. The adverse features observed at final pathology were separately analyzed and presented with respect to PI-RADSv2 subgroups.

\section{Statistical analysis}

The R program (version 3.2.3, the $R$ foundation for Statistical Computing, www.r-project.org) using epiR and $\mathrm{pROC}$ packages was used to perform statistical analysis. Descriptive statistics are reported as mean \pm standard deviation. Diagnostic performance was expressed with sensitivity, specificity, and positive and negative predictive values. The histopathological report was regarded as a reference standard. The $\chi^{2}$ test and Fisher's exact test were performed when appropriate. The significance level was set to $p<0.05$. The DeLong test was used to compare areas under the curve $(A \cup C)$ of receiver operating characteristics (ROC) of staging models to reveal differences in the diagnostic performance. The Youden index method was used to determine the best threshold. Recognizing the fact that in daily clinical practice the decision regarding the extension of resection and NVB preservation is made separately for both sides of the prostate, two types of analysis were performed: per patient analysis for variables irrespective of sides; and per side analysis, in which both sides were considered as separate cases. Univariate and multivariate logistic regression models were performed to assess association of variables with EPE in per side analysis.

\section{Results}

\section{Clinical characteristics}

The main characteristics of 154 men included in our study are presented in Table I. Staging using mpMRI revealed EPE in 32 (20.8\%) men, while the tumor in the other $122(79.2 \%)$ men was assessed as organ confined (116 men, $75.3 \%$ ) or no lesion could be identified in the imaging (6 men, 3.9\%). In per patient analysis mpMRI had sensitivity of $30.2 \%$, specificity of $84.2 \%$, positive predictive value of $50.0 \%$ and negative predictive value of $69.7 \%$, with respect to EPE recognition.

\section{Detection of suspicious lesion with PI-RADS}

The PI-RADS scale was used to localize and assess lesions suspicious for harboring significant PCa, which is defined in PI-RADS guidelines as: GS $\geq 7$ and/or tumor volume $\geq 0.5 \mathrm{ml}$ and/or presence of EPE in histopathology (Table I). Overall, 262 lesions were identified in 148 men: one in 65 (42.2\%), two in $61(39.6 \%)$, three or more in 22 (14.3\%) patients. In $6(3.9 \%)$ men no lesion was detected. 
Table I. Clinical characteristics of 154 men included in the study

\begin{tabular}{|c|c|}
\hline Parameter & Value \\
\hline Age, mean \pm SD [years] & $63.7 \pm 6.17$ \\
\hline $\mathrm{PSA}$, mean $\pm \mathrm{SD}[\mathrm{ng} / \mathrm{ml}]$ & $10.7 \pm 13.2$ \\
\hline prostate volume, mean $\pm \mathrm{SD}[\mathrm{ml}]$ & $39.9 \pm 18.7$ \\
\hline $\mathrm{PSAD}$, mean $\pm \mathrm{SD}\left[\mathrm{ng} / \mathrm{ml}^{2}\right]$ & $0.31 \pm 0.46$ \\
\hline \multicolumn{2}{|l|}{ GS in biopsy: } \\
\hline$\leq 6$ & $56(36.3 \%)$ \\
\hline 7 & $74(48.1 \%)$ \\
\hline$\geq 8$ & $24(15.6 \%)$ \\
\hline \multicolumn{2}{|l|}{ CT: } \\
\hline CT1 & $57(37.0 \%)$ \\
\hline cT2 & $91(59.1 \%)$ \\
\hline$\geq \mathrm{cT3}$ & $6(3.9 \%)$ \\
\hline \multicolumn{2}{|l|}{ PI-RADS assessment category ${ }^{\dagger}$ : } \\
\hline $\mathrm{PI}-\mathrm{RADS} \leq 2$ & $89(28.9 \%)$ \\
\hline PI-RADS 3 & $44(14.3 \%)$ \\
\hline PI-RADS 4 & $108(35.1 \%)$ \\
\hline PI-RADS 5 & $67(21.7 \%)$ \\
\hline \multicolumn{2}{|l|}{ GS in histopathology: } \\
\hline$\leq 6$ & $49(31.8 \%)$ \\
\hline 7 & $84(54.6 \%)$ \\
\hline$\geq 8$ & $21(13.6 \%)$ \\
\hline \multicolumn{2}{|l|}{ pT: } \\
\hline$\leq \mathrm{pT} 2$ & $101(65.6 \%)$ \\
\hline$\geq \mathrm{pT3}$ & $53(34.4 \%)$ \\
\hline \multicolumn{2}{|l|}{ Surgical margins' status: } \\
\hline PSM - & $134(87.0 \%)$ \\
\hline PSM+ & $20(13.0 \%)$ \\
\hline
\end{tabular}

SD - standard deviation, PSA - prostate-specific antigen, PSAD - prostate specific antigen density, GS - Gleason score, CT - clinical stage in digital rectal examination, PI-RADS - Prostate Imaging Reporting and Data System, pT-pathologic stage, PSM - positive surgical margin. Unless otherwise specified all variables were calculated in per patient analysis. ${ }^{\dagger}$ Per side analysis.

\section{Comparison of staging performance}

Diagnostic performance of standard staging with mpMRI was compared to the PI-RADS scale using receiver operating characteristic curves (ROC) in per side analysis (Table II, Figure 1). mpMRI alone presented limited sensitivity in EPE detection (18.3\%), whereas PI-RADS achieved higher sensitivity (39.4\%) and slightly lower specificity ( $83.5 \%$ vs. $92 \%)$. The comparison of both staging methods using ROC curves revealed that PI-RADS significantly outperformed standard staging using mpMRI (AUC $=0.615$ vs. $0.552, p=0.036$ ). PI-RADS 5 was established as the best threshold for predicting EPE.

\section{Models for predicting side-specific EPE}

Univariate and multivariate regression models were created to select variables associated with side-specific EPE (Table III, Figure 2). In univariate models, all evaluated variables were significantly correlated with PCa stage. Stepwise regression using the leave-one-out method created a multivariable model, in which only PI-RADS, PSA and GS in biopsy remained significant variables. Of note, PI-RADS

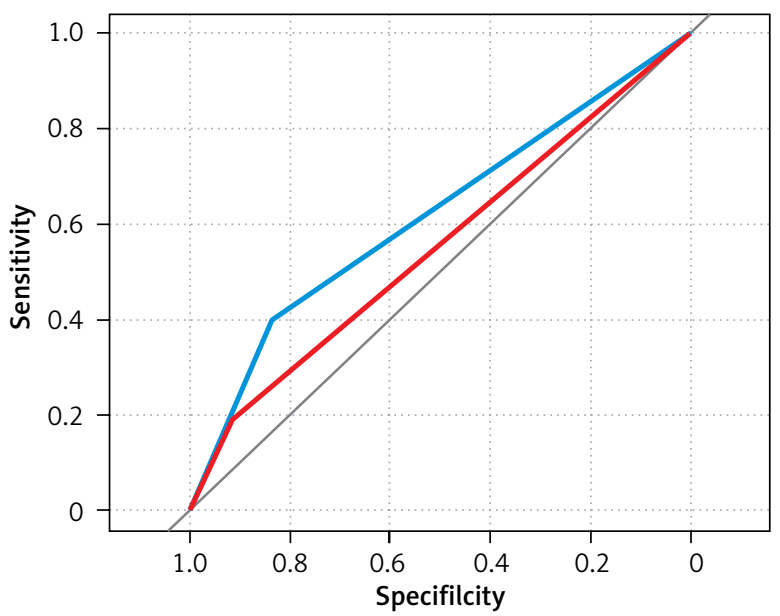

Figure 1. Comparison of staging performance of mpMRI and PI-RADS using ROC

PI-RADS is shown as the blue curve, mpMRI as the red curve.

Table II. Staging performance of $\mathrm{mpMR}$ and PI-RADS ${ }^{\dagger}$

\begin{tabular}{|lcccccc|}
\hline Staging method & AUC $^{*}$ & Threshold & SE (\%) & SP (\%) & PPV (\%) & NPV (\%) \\
\hline PI-RADS & 0.615 & PI-RADS 5 & 39.4 & 83.5 & 41.8 & 82.2 \\
\hline mpMRI & 0.552 & iT2C/T3 & 18.3 & 92.0 & 40.6 & 79.0 \\
\hline
\end{tabular}

mpMRI - multiparametric magnetic resonance imaging, PI-RADS - Prostate Imaging Reporting and Data System, AUC - area under the curve, SE - sensitivity, $S P$ - specificity, PPV - positive predictive value, NPV - negative predictive value. ${ }^{\dagger} P e r$ side analysis. ${ }^{*}$ DeLong's test for two correlated $R O C$ curves: $p=0.0362$. 
Table III. Clinical variables associated with side-specific $\mathrm{EPE}^{\dagger}$

\begin{tabular}{|lcccc|}
\hline Parameter & \multicolumn{3}{c|}{ Analysis } \\
\cline { 2 - 5 } & \multicolumn{3}{c}{ Univariate } & Multivariate \\
\cline { 2 - 5 } & OR & $P$-value & OR & -value \\
\hline PSA & 1.04 & $0.0032^{* *}$ & 1.12 & $0.0081^{* *}$ \\
\hline PSAD & 2.30 & $0.0216^{*}$ & - & $0.0720^{\$}$ \\
\hline GS in biopsy & 1.07 & $0.0008^{* * *}$ & 1.72 & 0.8310 \\
\hline CT in DRE & 1.71 & $0.0483^{*}$ & 1.08 & $0.0038^{* *}$ \\
\hline PI-RADS $(\leq 4$ vs. 5$)$ & 3.31 & $0.0001^{* * *}$ & 3.40 & 0.9509 \\
\hline iT in mpMRI & 1.22 & $0.0126^{*}$ & 1.03 & \\
\hline
\end{tabular}

${ }^{5} p<0.1,{ }^{*} p<0.05,{ }^{* *} p<0.01,{ }^{* *} p<0.001$. Not included in the multivariate model because it is a combination of other variables. PCa - prostate cancer, $O R$ - odds ratio, PSA - prostate-specific antigen, PSAD - prostate-specific antigen density, DRE - digital rectal examination, PI-RADS - Prostate Imaging Reporting and Data System, $\mathrm{mpMRI}$ - multiparametric magnetic resonance imaging. ${ }^{\dagger}$ Per side analysis.

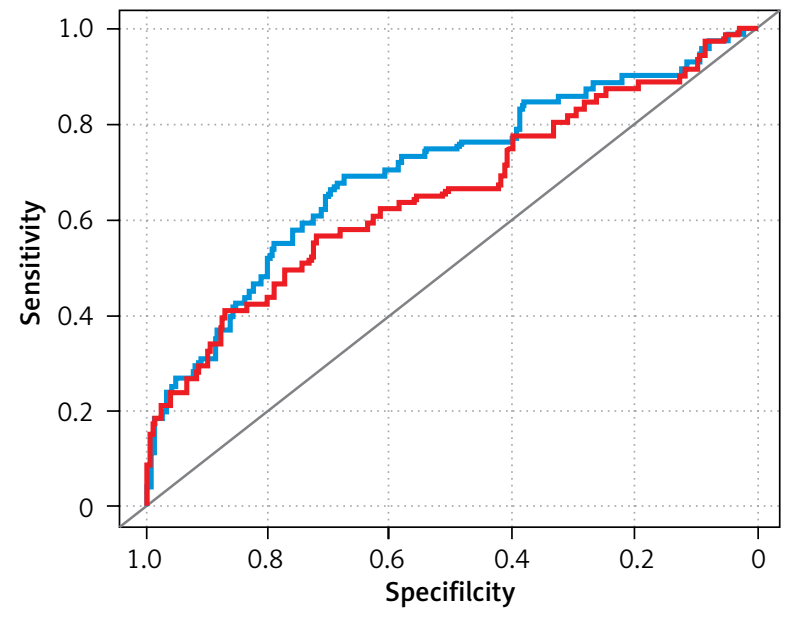

Figure 2. Comparison of staging performance of models incorporating mpMRI or PI-RADS using ROC

The model using PI-RADS is shown as the blue curve, the model using $\mathrm{mPMRl}$ as the red curve.

and PSA showed a strong correlation with PCa stage, whereas $\mathrm{mpMRI}$ stage did not.

\section{Surgical plan regarding NVB preservation}

Prior to mpMRI and according to EAU recommendations, NVB-sparing surgery was anticipated on $113(36.7 \%)$ sides, while on the remaining 195 $(63.3 \%)$ sides preservation would not be performed because of one or more unfavorable features: PSA $\geq 10 \mathrm{ng} / \mathrm{ml}$ (on 76 sides; 39.0\%), tumor palpable in DRE (on 75 sides; $38.5 \%$ ), GS $\geq 7$ in biopsy (on 95 sides; $48.7 \%)$.
The initial surgical plan regarding NVB preservation was modified on 96 (31.2\%) sides based on the mpMRI result, which was proper in 67 (69.8\%) cases. NVB sparing was performed on 85 (27.6\%) sides, on which NVB would have been resected if mpMRI had not been included. That modification was appropriate in 64 (75.3\%) cases. When the surgical plan was not modified, it was appropriate on 133 of 212 (62.7\%) sides.

When mpMRI prompted NVB resection (11 sides, $3.6 \%)$, it was appropriate in $3(27.3 \%)$ cases. The surgical plan was changed more frequently when no EPE on imaging or a lower PI-RADS sore was encountered (Table IV). Modification of the initial plan based on the mpMRI result using PI-RADS was not associated with higher risk of PSM.

\section{PI-RADS and local staging}

The prevalence of EPE and PSMs among different PI-RADS scores is presented in Table $\mathrm{V}$. Detection of PI-RADS 5 linked to the highest risk of EPE (41.8\%) and PSM (19.4\%) simultaneously. Noteworthy, NVBS in PI-RADS 3 were preserved as frequently as in PIRADS 4, despite the risk of EPE in PI-RADS 3 near to PI-RADS 5. The PSM rate was higher in PI-RADS 5 and PI-RADS 3 than in other scores.

\section{Discussion}

Our study provides new insight into the value of mpMRI with the PI-RADSv2 score in the decision-making process regarding the extent of resection during RP. The results indicate that PI-RADSv2 
Table IV. Modification of surgical plan related to EPE detection in mpMRI and PI-RADS score

\begin{tabular}{|c|c|c|c|}
\hline Srgical plan & Unchanged $(n=212)$ & Changed $(n=96)$ & $P$-value \\
\hline PI-RADS: & & & $0.0277^{\star}$ \\
\hline$\leq 4$ & $158(75.5 \%)$ & $83(86.5 \%)$ & \\
\hline 5 & $54(25.5 \%)$ & $13(13.5 \%)$ & \\
\hline iT in mpMRI: & & & $0.0167^{\star}$ \\
\hline EPE - & $182(85.8 \%)$ & 92 (95.8\%) & \\
\hline $\mathrm{EPE}+$ & $30(14.2 \%)$ & $4(4.2 \%)$ & \\
\hline NVB: & & & $<0.001^{\star \star *}$ \\
\hline Preservation & $102(48.1 \%)$ & 85 (88.5\%) & \\
\hline Resection & $110(51.9 \%)$ & 11 (11.5\%) & \\
\hline pT: & & & 0.8540 \\
\hline$\leq \mathrm{pT} 2$ & $162(76.4 \%)$ & 75 (78.1\%) & \\
\hline$\geq \mathrm{pT3}$ & $50(23.6 \%)$ & $21(21.9 \%)$ & \\
\hline Surgical margin: & & & 0.1960 \\
\hline PSM - & $187(88.2 \%)$ & $90(93.8 \%)$ & \\
\hline$\overline{P S M}+$ & $25(11.8 \%)$ & $6(6.2 \%)$ & \\
\hline
\end{tabular}

Table V. PI-RADS score related to local staging and surgical margins status ${ }^{\dagger}$

\begin{tabular}{|c|c|c|c|c|}
\hline Parameter & $\begin{array}{c}\text { PI-RADS } \leq 2 \\
(n=89)\end{array}$ & $\begin{array}{l}\text { PI-RADS } 3 \\
(n=44)\end{array}$ & $\begin{array}{l}\text { PI-RADS } 4 \\
(n=108)\end{array}$ & $\begin{array}{c}\text { PI-RADS } 5 \\
(n=67)\end{array}$ \\
\hline \multicolumn{5}{|l|}{$\mathrm{pT}^{\star \star \star *}:$} \\
\hline pT2 & 72 (80.9\%) & 29 (65.9\%) & 97 (89.8\%) & 39 (58.2\%) \\
\hline pT3 & 17 (19.1\%) & 15 (34.1\%) & $11(10.2 \%)$ & 28 (41.8\%) \\
\hline \multicolumn{5}{|l|}{$\mathrm{PSM}^{\star *}:$} \\
\hline PSM - & 82 (92.1\%) & 37 (84.1\%) & $104(96.3 \%)$ & $54(80.6 \%)$ \\
\hline PSM + & 7 (7.9\%) & 7 (15.9\%) & $4(3.7 \%)$ & 13 (19.4\%) \\
\hline \multicolumn{5}{|l|}{$N V B^{\star \star \star}:$} \\
\hline Preservation & 75 (84.3\%) & 27 (61.4\%) & 67 (62.0\%) & 18 (26.9\%) \\
\hline Resection & $14(15.7 \%)$ & 17 (38.6\%) & 41 (38.0\%) & 49 (73.1\%) \\
\hline
\end{tabular}

${ }^{{ }^{*}} p<0.05,{ }^{* *} p<0.01,{ }^{* * *} p<0.001$. PI-RADS - Prostate Imaging Reporting and Data System, $p T$ - pathological tumor staging, PSM - positive surgical margin. tper side analysis.

outperforms standard PCa staging with mpMRI by better prediction of side-specific EPE. With PI-RADS 5 as the best threshold, locally advanced PCa is over three times more frequent than in lesions with lower PI-RADS scores. The mpMRI result with $\mathrm{PI}$ RADS prompts adaptation of the extent of resection during RP in one-third of the cases, mostly toward more preserving NVB surgery. The decision based on PI-RADS does not increase the risk of PSMs.
In our study mpMRI had moderate diagnostic sensitivity (30.2\%) and specificity (84.2\%) in detecting EPE, which is slightly lower than in the recently published meta-analysis, where corresponding values were $61 \%$ and $88 \%$, accordingly [1]. However, the prevalence of EPE was rather high in our cohort (34.4\%), which means that the meta-analysis results may not be easily extrapolated to our study [1]. Moreover, diagnostic performance of mpMRI dropped 
drastically, when per side analysis was implemented. Rud et al. first drew attention to such inconsistency in the results, revealing a decrease in accuracy from $69 \%$ to $57 \%$ when side-specific EPE was taken into account [13]. In our opinion, simplified per-patient analysis of EPE detection performed in most studies does not completely reflect clinical practice, in which precise determination of side infiltrated by the tumor is crucial for planning of NVB-sparing surgery or focal therapy. This phenomenon may partially explain some controversial findings in which $\mathrm{mpMRI}$ false negative for EPE prompting a surgeon to perform NVB-sparing surgery does not necessarily lead to PSM [14].

Imperfect staging accuracy of MRI may result from limited ability to recognize microscopic infiltration of the prostate capsule [1, 2]. Considering extensive and focal ECE separately, sensitivity of mpMRI decreases drastically from $62.5 \%$ to $14.3 \%$ [2]. By relying only on direct radiological signs, for instance macroscopic periprostatic infiltration by the tumor, the reader often fails to detect microscopic EPE; therefore indirect signs strongly suggesting EPE are also needed. These include, for example, capsule irregularity, NVB thickening and recto-prostatic angle obliteration, among many others described in the literature. An attempt was made to regulate this issue by providing a separate table for assessing probability of EPE in ESUR prostate MRI guidelines [5]. Despite giving promising results [15], systematic assessment of EPE by points did not gain broader acceptance. In fact, it was later abandoned in reissued PI-RADSv2 recommendations, where the likelihood of EPE is assessed in a descriptive manner giving a binary solution [6].

However, PI-RADSv2 is not a comprehensive tool and is designed mainly for detection, localization and description of lesions. We decided to evaluate its usefulness in predicting EPE. Interestingly, the PI-RADSv2 score was proved to be superior to standard staging using $\mathrm{mpMRI}$ in our cohort in per side analysis. With the best cutoff of 5 for predicting side-specific EPE, PI-RADSv2 had higher sensitivity than standard staging with mpMRI (39.4\% vs. $18.3 \%$ ) with slightly lower specificity (83.5\% vs. 92.0\%; Table II, Figure 1). Lim et al. presented similar findings by comparing PI-RADS scores 4 and 5 . The group revealed that PI-RADS 5 was linked to greater risk of EPE and the condition of lesion size $\geq 15 \mathrm{~mm}$ outperformed subjective assessment of EPE [16].
Furthermore, subjective impression of EPE was rarely a factor that increased the PI-RADS score, because in the vast majority of cases the tumor size was greater than $15 \mathrm{~mm}$. Therefore, Lim et al. concluded that inter-reader reproducibility may be improved by removing such descriptive terms from PI-RADSv2 [16]. In our study, EPE occurred four times more often when a PI-RADS 5 lesion was detected than PI-RADS 4 (41.8\% vs. $10.2 \%$; Table V). Radiological hallmarks that discriminate these two entities are: $\geq 15 \mathrm{~mm}$ in the greatest dimension and/or the presence of definite EPE. Of note, both these features are strongly linked to each other: the greater the dimension of the tumor in MRI, the broader the tumor-capsule contact length is, which in turn strongly correlates with microscopic ECE [17]. By implementing this indirect sign of locally advanced disease, inter-reader agreement and sensitivity for EPE may be improved [18]. Moreover, comparing PI-RADSv1 to PI-RADSv2, the latter allows one to reduce understaging more efficiently, and supposedly would bring greater benefit in planning NVB sparing [19].

The relevance of size may also explain a higher prevalence of EPE in PI-RADS 3 than in PI-RADS 4 lesions in our cohort (34.1\% vs. $10.2 \%$, Table V). PIRADS 3 cases, which turned out to be pT3 tumors, were mostly large lesions located in the peripheral zone, which, however, did not meet the criteria of PIRADS 5, such as evident restriction of diffusion or invasive behavior, or presented no focal enhancement. PI-RADS 3 remains a challenging entity, because of overlapping radiologic signs of benign and malignant conditions within such a lesion, with the risk of clinically significant PCa being equivocal. Only one in five men with PI-RADS 3 lesions would presumptively harbor clinically significant PCa [20]. Noteworthy, some refinements have already been proposed for upgrading the PI-RADS 3 score under several conditions, with lesion size $\geq 10 \mathrm{~mm}$ [21].

Another alarming finding was that EPE was also present on sides where PI-RADS indicated low or very low likelihood of clinically significant PCa (19\%; Table V). Locally advanced disease found on the side with PI-RADS score $\leq 2$ may simply result either from misinterpretation of images or contralateral large lesion extension into the seemingly unaffected side, causing EPE on both sides. A meta-analysis of studies addressing the diagnostic accuracy of RI-RADSv2 revealed high sensitivity of $89 \%$ and specificity of $73 \%$ for PCa detection [22, 23]; however, only a minority of 
the included studies used a postoperative specimen as a reference standard. On the other hand, Reisaeter et al. revealed, by doing region-by-region analysis [24], that the reliability of PI-RADS in precise localization of index lesions is eventually much lower than anticipated. Three readers managed to precisely identify only around one-half of the tumor extent [24].

The dilemmas described above touch upon an issue of the role of MRI in NVB preservation in patients subjected to RP. Following EAU recommendations, NVB-sparing surgery would be performed on $36.7 \%$ of sides in our study. However, after including $\mathrm{mpMRI}$, that initial surgical plan was modified in one-third of cases (31.2\%), which turned out to be correct in $69.8 \%$. These results are comparable with the study of Schiavina et al. [3], where the MRI result appeared to be even more influential and changed the NVB-sparing plan in over half of cases $(56.2 \%)$ in per side analysis with overall appropriateness of $75.3 \%$. The decision was changed even-handedly to more preserving or a more radical approach in that study, whereas our results indicated that NVB sparing was chosen nine times more frequently than resection ( $89 \%$ vs. $11 \%$ ). The differences are probably caused by adopting other initial criteria for NVB preservation. Nevertheless, these findings underline the influential role of MRI in NVB preservation. Hara et al. developed a multivariate model, in which a cancer focus with a capsular contact or ECE on DWI was a significant predictor of ECE [25], which in combination with biopsy findings resulted in high negative predictive value (93.9\%) in excluding side-specific ECE. Similar findings were reproduced in the study focusing on posterolateral regions of the gland, where MRI has also been proved to be an independent predictive factor for ECE [26].

Misleading information delivered by MRI, which results in more preserving NVB sparing, might virtually cause PSM. Indeed, the PSM and EPE rates in PI-RADS 3 and PI-RADS 5 lesions are similar in our cohort and higher than in PI-RADS 4 (Table V). However, the overall PSM rate remains similar, regardless of whether the decision on the extent of resection was changed or not (6.2\% vs. $11.8 \%$; $p=0.196)$. In a randomized controlled trial, despite preoperative $M R I$, the overall risk for PSM (23\% in non-MRI vs. $19 \%$ in MRI group) was not changed. Subgroup analysis has shown the benefit of imaging in patients with CT1C PCa, in whom a relative reduction of PSM by $41 \%$ was observed [27]. In contrast, in the study with a matched non-MRI control group, the PSM rate was twice as high as in the group with preoperative MRI (24.1\% vs. $12.4 \%$ ) [3]. These mediocre results mean that, in spite of assisting in surgical planning, the capacity of MRI to decrease PSM rates remains unconfirmed. Another innovative approach to improve the outcomes of MRI-guided surgery and reduce the PSM rate, yet minimize NVB removal, is intraoperative frozen section analysis (IFS). Petralia et al. revealed that IFS directed toward MRI detected lesions in patients subjected to nerve-sparing robot-assisted RP was able to halve PSM rates when compared to well-matched controls who underwent surgery only [28]. However, despite several retrospective studies demonstrating an average decrease of $13.2 \%$ in PSM rates, the use of IFS is still a matter of debate. It is time consuming and costly. Furthermore, this technique is prone to artifacts and sampling error that limit its sensitivity and specificity in the detection of positive surgical margins [29].

A few limitations of our study need to be acknowledged. First, this is non-randomized study and well-designed prospective trials are needed to confirm our findings. Second, the radiologist was aware of clinical characteristics of included patients, which could inadvertently influence the interpretation of mpMRI and PI-RADS score assignment. Third, the study was based on a single radiological assessment, thereby reflecting everyday clinical practice. Moreover, our criteria of NVB preservation, based on EAU recommendations, may not fully reflect the standards in other centers; therefore our results cannot be easily generalized.

\section{Conclusions}

The PI-RADS score is more accurate in predicting side-specific EPE than standard PCa staging with $\mathrm{mpMRI}$ and may assist in the decision-making process regarding the extent of resection during ERP. The mpMRI result with PI-RADSv2 prompts the adaptation of an NVB preservation plan in one-third of cases, mostly toward a more preserving NVB-sparing approach. It appears to leave the risk of PSM unchanged. PI-RADSv2 may be incorporated into the preoperative workup of men undergoing RP, but refinements are needed.

\section{Conflict of interest}

The authors declare no conflict of interest. 


\section{References}

1. de Rooij M, Hamoen EHJ, Witjes JA, et al. Accuracy of magnetic resonance imaging for local staging of prostate cancer: a diagnostic meta-analysis. Eur Urol 2016; 70: 233-45.

2. Feng TS, Sharif-Afshar AR, Smith SC, et al. Multiparametric magnetic resonance imaging localizes established extracapsular extension of prostate cancer. Urol Oncol: Semin Ori Investig 2015; 33: 109.e15-22.

3. Schiavina R, Bianchi L, Borghesi M, et al. MRI displays the prostatic cancer anatomy and improves the bundles management before robot assisted radical prostatectomy. J Endourol 2018; 32: 315-21.

4. Nguyen LN, Head L, Witiuk K, et al. The risks and benefits of cavernous neurovascular bundle sparing during radical prostatectomy: a systematic review and meta-analysis. J Urol 2017; 198: 760-9.

5. Barentsz JO, Richenberg J, Clements R, et al. ESUR prostate MR guidelines 2012. Eur Radiol 2012; 22: 746-57.

6. Weinreb JC, Barentsz JO, Choyke PL, et al. PI-RADS Prostate Imaging - Reporting and Data System: 2015, Version 2. Eur Urol 2016; 69: 16-40.

7. Park SY, Cho NH, Jung DC, et al. Prostate imaging-reporting and data system version 2: beyond prostate cancer detection. Korean J Radiol 2018; 19: 193-200.

8. De Cobelli O, Terracciano D, Tagliabue E, et al. Predicting pathological features at radical prostatectomy in patients with prostate cancer eligible for active surveillance by multiparametric magnetic resonance imaging. PLoS One 2015; 10: 1-14.

9. Matsuoka Y, Ishioka J, Tanaka H, et al. Impact of the prostate imaging reporting and data system, version 2, on MRI diagnosis for extracapsular extension of prostate cancer. Am J Roentgenol 2017; 209: W76-84.

10. Kozikowski M, Powroźnik J, Malewski W, et al. 3.0-T multiparametric MRI modifies the template of endoscopic, conventional radical prostatectomy in all cancer risk categories. Arch Med Sci 2018; 14: 1387-93.

11. Dobruch J, Piotrowicz S, Skrzypczyk M, et al. Clinical value of extended pelvic lymph node dissection in patients subjected to radical prostatectomy. Videosurgery Miniinv 2014; 9: 64-70.

12. Briganti A, Larcher A, Abdollah F, et al. Updated nomogram predicting lymph node invasion in patients with prostate cancer undergoing extended pelvic lymph node dissection: the essential importance of percentage of positive cores. Eur Urol 2012; 61: 480-7.

13. Rud E, Klotz D, Rennesund K, et al. Preoperative magnetic resonance imaging for detecting uni- and bilateral extraprostatic disease in patients with prostate cancer. World J Urol 2015; 33: 1015-21.

14. Billing A, Buchner A, Stief C, et al. Preoperative mp-MRI of the prostate provides little information about staging of prostate carcinoma in daily clinical practice. World J Urol 2015; 33: 923-8.

15. Boesen L, Chabanova E, Logager V, et al. Prostate cancer staging with extracapsular extension risk scoring using multiparametric MRI: a correlation with histopathology. Eur Radiol 2015; 25: 1776-85.

16. Lim CS, McInnes MDF, Lim RS, et al. Prognostic value of prostate imaging and Data Reporting System (PI-RADS) v. 2 assessment categories 4 and 5 compared to histopathological outcomes after radical prostatectomy. J Magn Reson Imaging 2016; 46: 257-66.

17. Baco E, Rud E, Vlatkovic L, et al. Predictive value of magnetic resonance imaging determined tumor contact length for extracapsular extension of prostate cancer. J Urol 2015; 193: 466-72.

18. Rosenkrantz AB, Shanbhogue AK, Wang A, et al. Length of capsular contact for diagnosing extraprostatic extension on prostate MRI: assessment at an optimal threshold. J Magn Reson Imaging 2016; 43: 990-7.

19. Matsuoka Y, Ishioka J, Tanaka H, et al. Impact of the prostate imaging reporting and data system, version 2, on MRI diagnosis for extracapsular extension of prostate cancer. Am J Roentgenol 2017; 209: W76-84.

20. Schoots IG. MRI in early prostate cancer detection: how to manage indeterminate or equivocal PI-RADS 3 lesions? Transl Androl Urol 2018; 7: 70-82.

21. Rosenkrantz AB, Babb JS, Taneja SS, et al. Proposed adjustments to PI-RADS version 2 decision rules: impact on prostate cancer detection. Radiology 2017; 283: 119-29.

22. Hamoen EHJ, De Rooij M, Witjes JA, et al. Use of the prostate imaging reporting and data system (PI-RADS) for prostate cancer detection with multiparametric magnetic resonance imaging: a diagnostic meta-analysis. Eur Urol 2015; 67: 1112-21.

23. Woo S, Suh CH, Kim SY, et al. Diagnostic performance of prostate imaging reporting and data system version 2 for detection of prostate cancer: a systematic review and diagnostic metaanalysis. Eur Urol 2017; 72: 177-88.

24. Reisæter LA, Fütterer JJ, Halvorsen OJ, et al. 1.5-T multiparametric MRI using PI-RADS: a region by region analysis to localize the index-tumor of prostate cancer in patients undergoing prostatectomy. Acta Radiol 2015; 56: 500-11.

25. Hara T, Nakanishi H, Nakagawa T, et al. Ability of preoperative 3.0-Tesla magnetic resonance imaging to predict the absence of side-specific extracapsular extension of prostate cancer. Int J Urol 2013; 20: 993-9.

26. Pak S, Park S, Ryu J, et al. Preoperative factors predictive of posterolateral extracapsular extension after radical prostatectomy. Korean J Urol 2013; 54: 824-9.

27. Rud E, Baco E, Klotz D, et al. Does preoperative magnetic resonance imaging reduce the rate of positive surgical margins at radical prostatectomy in a randomised clinical trial? Eur Urol 2015; 68: 487-96.

28. Petralia G, Musi G, Padhani AR, et al. Robot-assisted radical prostatectomy: multiparametric MR imaging-directed intraoperative frozen-section analysis to reduce the rate of positive surgical margins. Radiology 2015; 274: 434-44.

29. Nunez AL, Giannico GA, Mukhtar F, et al. Frozen section evaluation of margins in radical prostatectomy specimens: a contemporary study and literature review. Ann Diagn Pathol 2016; 24: 11-8.

Received: 28.01.2019, accepted: 16.02.2019. 